\title{
An Indoor Hybrid Localization Approach Based on Signal Propagation Model and Fingerprinting
}

\author{
Junhuai $\mathrm{Li}^{1}$, Bo Zhang ${ }^{1}$, Hailing $\mathrm{Liu}^{2}$, Lei $\mathrm{Yu}^{1}$ and Zhixiao Wang ${ }^{1}$ \\ ${ }^{1}$ School of Computer Science \& Engineering,Xi'an University of Technology, \\ $X i$ 'an 710048,China \\ ${ }^{2}$ College of Electronic Information Engineering,Chongqing University of Science and \\ Technology,Chongqing 400050,China. \\ junhuai.li@gmail.com.zhangboxaut@163.com
}

\begin{abstract}
RFID-based location awareness is becoming the most important issue in many fields in recent years, such as ubiquitous computing, mobile computing. In indoor localization systems RSSI-based methods are usually used in office buildings. However, RSSI is susceptible to external influences, and performances unstably due to the environmental factors affecting signal propagation. In this paper, we propose a new hybrid localization method for tracking moving object using the two typical methods which are signal propagation model and fingerprinting. According to a threshold which is defined as an effective working distance of signal propagation model between target tag and RFID reader, we choose the different localization algorithm to estimate the location of moving object. The threshold is obtained by calculating the slop of signal attenuation curve. If the distance is within the effective reading range of RFID reader, we revise signal propagation model by maximum likelihood estimation and use it to calculate the object position by minimum cumulative error. Otherwise, the fingerprinting location method is used in the external area, and the particle filter is also used as the core algorithm. The experimental results show that our method not only reduces the computation complexity but also ensures the accuracy in large indoor area.
\end{abstract}

Keywords: Indoor localization, signal propagation model, fingerprinting location, RFID

\section{Introduction}

With the development of wireless technology, the location-based service (LBS) gradually becomes a hot research field in recent years. One of the most well-known location systems is the GPS which is widely used in many fields for tracking moving objects located outdoors. However, GPS does not perform very well and even causes larger errors inside buildings for the signal is susceptible [1]. Therefore, indoor positioning system has attracted growing attention in these days.

Nowadays, some typical location systems and approaches have been proposed, such as infrared [2], ultrasonic wave [3] and RF [4-6] etc. Based on the RSSI (Received Signal Strength Indicator), there have been various techniques developed which can be broadly divided into two categories: signal propagation model and fingerprinting. The advantage of signal propagation model is simple, and it shows the relationship between signal and position. However, its disadvantage is also obvious, and the biggest challenge is accuracy and limited effective distance. On the other hand, fingerprinting is more accurate, but it requires a huge database to store fine-grained location data in addition to exhaustive search to locate suitable 
fingerprint match. Some new methods have been introduced into fingerprinting location methods in recent years, such as particle filter and decomposition of the graph [7-9].

In past years, many RF-based indoor location systems has been developed, LANDMARC [10] and RADAR [11] are two most well-known systems. LANDMARC system has been a foundation for many RFID-based solutions and it introduces the reference tags to estimate the location of moving object. In the process of location, it firstly records the received RSSI of tracking object, and then chooses $k$ reference tags by the $k$-nearest algorithm. Since the coordinate of reference tag is predetermined, so LANDMARC system uses the position information of $k$ neighbor reference tags to estimate the coordinate of target object. RADAR is an early approach using RSSI to establish an indoor positioning system, which combines the empirical fingerprinting method and theory propagation model to track an object. In order to reduce the complexity in collecting the RSSI data, the RADAR system improves the original signal propagation model and constructs the radio map based on the improved model.

Signal propagation model and fingerprinting are two typical location approaches nowadays. Location based on signal propagation model is simple and has less computation. However, in order to get higher accuracy, an accurate model should be constructed to reflect the real indoor environment. Moreover, the effective distance of signal propagation model is limited to tens meters or less. Once the distance is more than the effective working distance of RFID reader, the correlation between distance and RSSI becomes weak, and the location errors increase accordingly. Compared with the approach of signal propagation model, fingerprint location is relatively accurate, but it's always with much computation and high complexity. Based on fingerprinting approach, it is necessary to collect much information of sample points in the location area. Once the environment changed, and then the previous collected information will be invalid. Although some methods have been proposed to solve these problems, the complexity is still very high, especially in large location area.

In this paper, we propose a new hybrid location approach. It can dynamically switch the location strategy according to a threshold which is defined as an effective working distance of signal propagation model between target tag and localization reader. The threshold is obtained by calculating the slop of signal attenuation curve. If the distance is within the effective reading range of RFID reader, we revise the signal propagation model by maximum likelihood estimation and use it to calculate the object position by minimum cumulative error. As the distance increases, signal propagation model becomes inaccurate and then fingerprinting-based location model will be used. And this method includes offline and online phases. In order to solve the uncertainty problem of fingerprinting-based location method, many statistic approaches have been proposed and the most important one is particle filter algorithm. Particle filter is a Bayesian method which uses many particles to estimate the probability density of the tracking object, and many papers have proven that it has high location accuracy. So we use this method in the online phrase.

The rest of this paper is organized as follows. In the next section, we introduce the hybrid location approach. The new location approach will be separated into three parts to illustrate in Section 2.1, Section 2.2 and Section 2.3 respectively. Some experiments are conducted and the results are shown in Section 3. Finally, in Section 4, we conclude the paper and list our future work.

\section{Hybrid Location Approach}

The signal propagation model is simple to implement and its main idea is to construct the relationship between RSSI and distance. However, the distance from the RSSI in signal propagation model is susceptible to multi-path reflections, diffraction and scattering of signal. 
The fingerprinting model has high positioning accuracy. Although this model is effective, its main drawback is that the database needs rebuilding when location environment changes. In this paper, a hybrid modeling is proposed. It combines the simple of propagation modeling and high positioning accuracy of fingerprinting model.

We have known that the effective distance of signal propagation model has a threshold $\left(d_{t h}\right)$. If the distance from a reader to tracking object is less than $d_{t h}$, the correlation between RSSI and transmission distance is obvious. Accordingly, the estimated value of distance is closer to the real distance and the location error is smaller. However, when the distance is larger than $d_{t h}$, signal propagation model is no longer profitable. Here $d_{t h}$ is defined as the critical value of signal propagation model, and it is determined by the accuracy requirements of location system.

In a large location area, it can be divided into two parts: interior area and external area. In the interior area, the received RSSI by reader meets the signal propagation model. However, if a tag appears in the external area which is far away from some readers, the correlation calculated from the model declines. Although tag and reader can communicate with each other in the external area, the limitation of signal propagation model becomes obvious. Thus, choosing proper location strategy has large influence on the accuracy.

As is shown in Figure 1, localization environment is composed of the three areas: interior, interim and exterior. Where $K$ and $S$ represent the distance between the reader and the edge of external region. $D$ and $T$ are the readers' effective radiuses which are obtained by the signal propagation model, and the edges of the interior area are composed of these radiuses. On the path $k$ from the reader $R_{i}$ to the border of the external region, if the object is within the range $T$ (interior region), location based on signal propagation model is effective. In the external region, however, it is no longer applicable, because there is at least one reader to receive invalid RSSI. In this case, if signal propagation model method is still used, then the location error will be larger. So our basic idea is to change the location strategy and use fingerprinting approach to estimate the location of moving object in this paper. Especially, the way above is able to obtain good location results when the location environment is complex and the object appears near the edge of the external area.

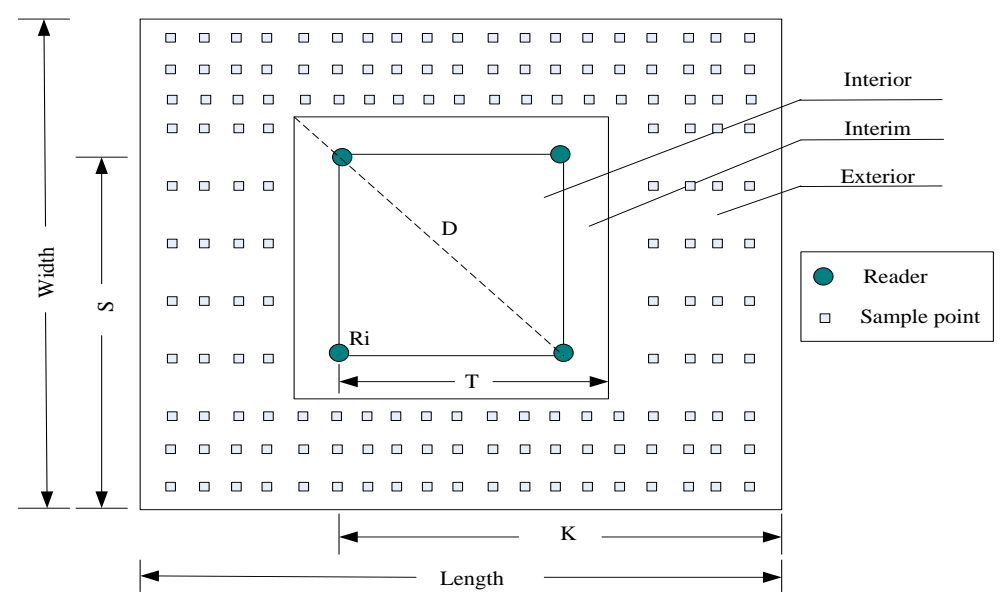

Figure 1. Localization Environment Analysis

\subsection{The Signal Propagation Mode}

2.1.1. The Correction of the Signal Propagation Model: Signal propagation model location is based on signal strength indication (RSSI). The model uses the concept of signal strength 
loss with distance to estimate the position of moving object. As the position of receiving device is known, so it is easy to compute the distance between the moving object and signal receiver. Signal is influenced by many factors in the propagation process. These factors play an important role on the relationship between signal strength and distance. Therefore, shadowing factor $\xi$ should be added to the propagation model to enhance the accuracy of this model. Shadowing factor is a variable in signal propagation model, which reflects the change of signal with environment in the propagation process. Thus we can obtain the signal strength at the distance $d$ by using the following equation (Eq.(1)):

$$
\overline{P L}(d)=\mathrm{P}_{\mathrm{t}}-\overline{P L}\left(d_{0}\right)-10 n \lg \frac{d}{d_{0}}-\xi
$$

Where $\overline{P L}\left(d_{0}\right)$ is the mean signal loss of signal transmitter at perigee $d_{0}$, and $P_{t}$ is the initial transmission power of tag. $n$ represents the signal loss index, it is concerned with the specific location environment, and it only can be tested in corresponding environments. Through intensive experiments, it is found that the value of $n$ is generally around 3. Even if the readers and tags are different, $n$ between different readers and tags is close. The shadowing factor obeys a normal distribution, but in previous works, many location approaches have ignored the influence of $\xi$. In this paper, our hybrid location method needs an accurate border value of signal propagation model. Therefore, it is necessary to get a more accurate model. To estimate $\xi$ accurately, a lot of experiments should be conducted, which makes it time consuming. In order to reduce the complexity, we use the maximum likelihood function to estimate it.

Let $\xi$ be a random variable of continuous type, and it follows Gaussian distribution $\xi \sim \mathrm{N}\left(\mu, \sigma^{2}\right)$. In order to construct the distribution function, we conduct sampling experiments for $k$ times in location area. The reader $R_{i}$ and tag $T_{j}$ are chosen as the sampling facilities. The received RSSI and position information are recorded at each time. Then we can obtain $k$ samples $\xi_{1}, \xi_{2}, \xi_{3} \ldots \ldots . \xi_{k}$ by Eq. (1). So the likelihood function $\mathrm{L}\left(\mu, \sigma^{2}\right)$ with $\xi$ is as follows:

$$
\mathrm{L}\left(\mu, \sigma^{2}\right)=\prod_{\mathrm{i}=1}^{\mathrm{k}} \frac{1}{\sqrt{2 \pi} \sigma} e^{-\frac{\left(\xi_{i}-\mu\right)^{2}}{2 \sigma^{2}}}
$$

By calculating the partial derivative of function $\mathrm{L}\left(\mu, \sigma^{2}\right)$ on $\mu$ and $\sigma$, we can obtain:

$$
\hat{\mu}=\frac{1}{k} \sum_{i=1}^{k} \xi_{i}=\bar{\xi} \quad \hat{\sigma}^{2}=\frac{1}{k} \sum_{i=1}^{k}\left(\xi_{i}-\bar{\xi}\right)^{2}
$$

Where it's obvious that $\mu$ is approximately equal to the sample mean value of $\xi$.If the sample points are enough, the estimated value $\hat{\mu}$ can reflect the feature of $\xi$ in the specific environment.

To evaluate the performance of the estimated model, we conduct sampling experiments every $100 \mathrm{~cm}$ in the location environment [0-30m], and repeat 50 times for each point. After filtering the wrong data, we record the maximum, minimum and mean value of RSSI respectively. Figure 2 shows a comparison of the experiment data from the predicting model. It 
also shows that our predicting model has the advantage of high accuracy. And when the distance is larger than $16 \mathrm{~m}$, the correlation declines and error begins to increase.

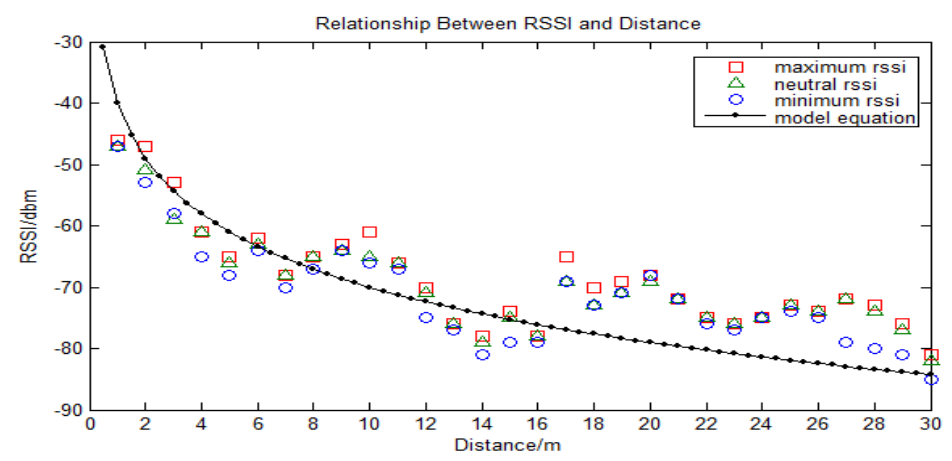

Figure 2. Signal Propagation Model Estimation

2.1.2. Location Approach Based on Signal Propagation Correction Model: Suppose that $k$ readers are deployed in location area, denoted as $R\left(R_{1}, R_{2}, R_{3} \ldots . . R_{k}\right)$, and each reader's coordinate is known. In the process of locating, readers and tags have the same setting, so RSSI at the perigee $d_{0}$ can be obtained by measuring. When tracking object with active tag enters into location area, $k$ readers will record their received $\mathrm{RSSI}_{i}$, and then we can calculate the distance $d_{i}$ between target tag and $R_{i}$ according to equation (4) as follows.

$$
d_{i}=d_{0} * 10 \frac{P_{t}-R S S I\left(d_{0}\right)-\xi-R S S I_{i}}{10 n}
$$

So we can get the distance vector between tags and readers, denoted as $\left[d_{1}, d_{2}, d_{3} \ldots . . d_{k}\right]$. Suppose the real coordinate of tracking object is $(x, y)$, and the objective function is defined as:

$$
\begin{gathered}
f(x, y)=\sum_{i=1}^{k}\left(d_{i d f}-d_{i}\right)^{2} \\
d_{i d f}=\sqrt{\left(x-R_{i x}\right)^{2}+\left(y-R_{i y}\right)^{2}}
\end{gathered}
$$

Where $f(x, y)$ represents the cumulative error between the true position and the predictive position. The value of $f(x, y)$ can largely reflects location accuracy. The smaller the value of function $f$ is, the higher the accuracy is. One of the methods to get the solution is to calculate the derivative on $x$ and $y$ respectively, and then make the derivative zero to get the minimum value. However, the derivative on $x$ and $y$ is complex and it's difficult to calculate the optimal solution. So, we use gradient descent method to solve the problem as equation (6) shown.

$$
H_{n+1}=H_{n}-\gamma T
$$

Where, $H_{n}$ is the coordinate in the $n$th iteration and $\gamma$ is the step length at every time. $T$ is the gradient direction of function $f$ at the initial point $\left(x_{0}, y_{0}\right)$. In order to simply the calculation, $\left(x_{0}, y_{0}\right)$ is set as the coordinate in the middle of all the readers and the iteration time should be less than 5 times.

The location approach above uses the mapping relationship between signal strength and the distance to locate. Meanwhile, the distance is also the main basis of location. Yet, the approach is limited in the following aspects: 
(1) It is influenced by the facilities. The main reason is that the approach is relative to the reader position, so the flexibility is limited. Moreover, the minimum distance error methods can be only used in the certain deployment.

(2) It needs more accurate models. Since distance is calculated directly by signal propagation model and signal strength changes in an exponential level when the distance is within the border value, it is necessary to conduct many experiments for signal propagation model.

(3) Gradient descent method can get the local optimal solution but not the best. Sometimes, it may cause extra error.

Although location results are restricted by the factors above, the advantage of this method is simple and the computational complexity is low. As long as the setting of the parameter is appropriate, a better location result can be obtained without other additional processing.

\subsection{Fingerprinting Location Approach}

When a target object appears in the external area, the accuracy of signal propagation model would decline sharply, and fingerprinting-based approach is the best way to conduct location in this area. Fingerprinting approach belongs to the category of pattern matching, and in general it is divided into offline and online phases. In the offline phase, the RSSI from different sample points are collected and stored in fingerprinting database called a radio map. In the online phrase, the readers collect RSSI of the target, and the RSSI is then compared with the sample data in the fingerprinting database. Finally, the target's coordinate can be estimated by the specific methods. In this paper, the particle filter algorithm is used to locate in the online phrase.

2.2.1. Offline Phrase: Data sampling is the main task of the offline phrase, which is a process of extracting the location space feature. In this paper, the external area is divided into a grid denoted as G, G can be thought as a $m^{*} n$ matrix, and each element $P_{i j}$ in the matrix represents a sample point. $P_{i j}$ is a triple $(x, y, s)$, where $x, y$ is the coordinate of the sample point in two-dimension space, $S$ is a vector group and $S=\left(S_{1}, S_{2}, S_{i} \ldots, S_{k}\right)$, where $S_{i}$ is the signal value received by reader $i$ from a sample point. Thus, the essence of location problem is a process which uses the information of $G$ and the observation value of target to estimate $(x, y)$.

The complexity of training phase depends on the number of sample points and sample time. In order to reduce complexity, many scholars have carried out some studies in this filed, and they found out that reducing the sample points and time have little influence on the location accuracy [12]. Therefore, if the location area is too large, it's feasible to reduce the complexity of sampling through some effective methods.

2.2.2. Location Phrase: Particle filter is a Bayesian estimation method. Unlike other methods, Particle filter has fewer requirements for the prediction equation and observation equation during the location phrase. What's more, the probability distributions of the particles are estimated via sampling, so it's suitable to solve the location problem.

Suppose the number of the initial particle is $N$, these particles stand for the probability distributions, denoted as $\left[P_{1}, P_{2}, \ldots \ldots . P_{N}\right]$. Every element in the vector group is a tuple $(L, S)$, where $L$ is the position and $S$ is the signal strength for each particle. All of these in $\left[P_{1}\right.$, $\left.P_{2}, \ldots \ldots . P_{N}\right]$ construct an exhaustive events group. Suppose that the tuple of the target is $\left(L_{o}, S_{o}\right)$, through the Bayesian formula, we can get equation (7) as follows: 


$$
p\left(\mathrm{~L}_{\mathrm{oi}} \mid S_{o i}\right)=\frac{p\left(S_{o i} \mid \mathrm{L}_{\mathrm{oi}}\right) p\left(\mathrm{~L}_{\mathrm{oi}}\right)}{\sum_{i=1}^{N} p\left(S_{o i} \mid \mathrm{L}_{\mathrm{oi}}\right) p\left(\mathrm{~L}_{\mathrm{oi}}\right)}
$$

Since the quantity of particles and the coordinates of selected particles are known, we can utilize the information of the particles to calculate their corresponding probabilities which will be regarded as the weight value after being processed.

(1) State transition function.

In general, the moving trajectory of tracking object is regular, so it's possible to use the historical trajectory to predict the future position. The state transition function is one of the most important aspects in the particle filter algorithm and its main function is to infer the information of the next state described as follows:

$$
x_{t}=f\left(x_{t-1}, u_{t}, w_{t}\right)
$$

Where $x_{t}$ is the particle's state at moment $\boldsymbol{t}, u_{t}$ is the control variation and $w_{t}$ represents model noise.

Literature [7] analyzes the influence of different state transition functions on the location result. In those functions, the $\mathrm{CPF}$ algorithm has a relatively better result, but it is still restricted when the number of particle is large. Literature [8] uses the Newton quadratic interpolation method to construct the state function and achieves a good result. The method is easy to construct and has a high precision, so it's also used as the state function $f$ in this paper. $f$ is shown as follows:

$$
\left\{\begin{array}{l}
x_{t}=x_{t-1}+v_{t} \cos \left(\alpha_{t}\right) \Delta t+n_{t} \\
y_{t}=y_{t-1}+v_{t} \sin \left(\alpha_{t}\right) \Delta t+n_{t}
\end{array}\right.
$$

Where $v_{t}$ is the moving speed of particle, its range is from 0.6 to 1.4. $\alpha_{t}$ is the moving direction and $n_{t}$ is the noise.

(2) The weight value of particle

We use the weight value of particles to estimate the influence on the location result. In equation (7), $p\left(\mathrm{~L}_{\mathrm{oi}} \mid S_{o i}\right)$ is one of formations of weight value. To obtain $p\left(\mathrm{~L}_{\mathrm{oi}} \mid S_{o i}\right)$, it's required to calculate the value of $p\left(S_{o i} \mid \mathrm{L}_{\mathrm{oi}}\right)$ We know that signal follows Gaussian distribution at each position in the location area, and the distribution function $\partial$ can be estimated by the fingerprinting data. In this way, the essence of getting the value of $p\left(S_{o i} \mid \mathrm{L}_{\mathrm{oi}}\right)$ is calculating the quadrature in the range of $\left(S_{o i}-\sigma, S_{o i}+\sigma\right)$ on function $\partial$.

Normalization is required after obtaining $N$ weights denoted as $W=\left[W_{1}, W_{2} \ldots W_{N}\right]$. Since the position of the target is regards as a kind of probability distribution, the summation of all the particle weights should be 1 . What's more, the weight reflects different influence intensity. Normalization can be achieved by the following formula:

$$
w_{i}=\frac{w_{i}}{\sum_{i=1}^{N} w_{i}}
$$

(3) Estimating object coordinates 
The weight vector group $W$ and the coordinate of each particle $L$ have been known. Therefore, we can obtain the final coordinates $(x, y)$ as follows:

$$
x=\sum_{i=1}^{N} w_{i} x_{i} \quad y=\sum_{i=1}^{N} w_{i} y_{i}
$$

Figure 3 shows the basic method to estimate the final coordinate by using the weight of each particle.

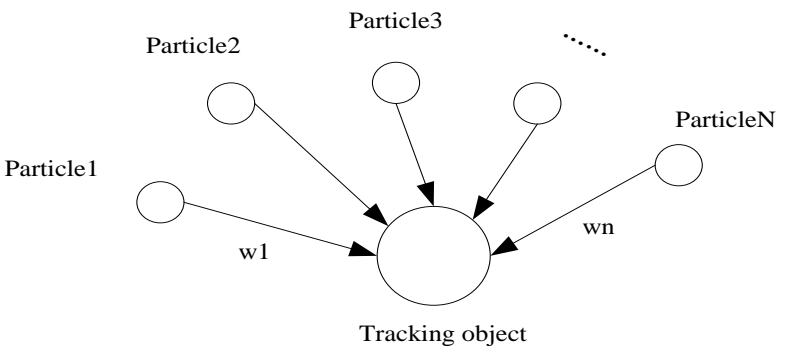

Figure 3. Estimating the Position by Particles

In summary, the algorithm of particle filter in this paper is described as follows:

Step 1 importance sample. Choosing $N$ particles from the fingerprinting database and determining initial weights for each one.

Step 2 Using the state transition equation to calculate the position of each particle in the next period as equation (9).

Step 3 Receiving the observed RSSI value of the target and calculating the posterior probability of each particle. Finally, normalizing the weight values of $N$ particles as equation (10).

Step 4 If the particle degradation phenomenon occurs, it's necessary to resample. The principle of resampling is that the particle with large weight will be copied and the small one must be thrown away.

Step 5 calculating the final position of the target through the weights and particle coordinates as equation (11).

\subsection{Determine the Border of the Propagation Model}

In our hybrid location approach, one of the most important tasks is to accurately estimate the border of signal propagation model. The border value is an important basis to determine which location approach should be selected. A simple and effective method to get the border value is described as follows:

Suppose the length of location area is within the range $[0, K)$ and the critical distance of signal propagation model is $T$. From equation (3), we found that when the distance is within ( 0 , $T$ ), the slope of the curve changes a lot with the distance, which means the correlation between RSSI and distance is obvious. But when the distance is within the range ( $T, K)$, it changes a little. Therefore, the effective distance $T$ can be estimated by calculating the slope of propagation model. Since the transmission power $P_{t}$ and power loss $P L\left(d_{0}\right)$ is constant, meanwhile, the shadowing factor $\xi$ can be obtained by the sample, we get the equation as follows:

$$
\operatorname{pl}(d)^{\prime}=\left(-10 n \lg \left(\frac{d}{d_{0}}\right)\right)^{\prime}=\frac{-10 \mathrm{n}}{\operatorname{In} 10^{*} \mathrm{~d}}
$$


When $d$ tends to be infinite $(d \rightarrow \infty)$, the slope is close to 0 . In order to determine the value of $T$, a threshold value TS about slope should be given. The value of TS is helpful to find the starting point when curve begins to flatten. Generally, $T S$ should be less than 1 . And then $T$ can be inferred by the following equation.

$$
\begin{array}{r}
\operatorname{pl}(d)^{\prime} \leq-T S \quad(\mathrm{TS}>0) \\
\Rightarrow \frac{-10 \mathrm{n}}{\operatorname{In} 10} * \frac{1}{d} \leq-\mathrm{TS} \\
\Rightarrow d \leq \frac{10 n}{(\operatorname{In} 10) T S}
\end{array}
$$

Therefore $\mathrm{T}=\frac{10 n}{(\operatorname{In} 10) T S}$. When $n=3.0, T S=0.5, T=26 \mathrm{~m}$, that is the effective border of propagation model is $26 \mathrm{~m}$.

Figure 4 illustrates the method above for determining the border. Location based on signal propagation model and fingerprinting can be accurately separated by $T$ value. From the definition of $T$, we know that $T$ is relative to loss exponent, measurement value of perigee and tangential threshold. With different location environments and sampling points, $n$ is likely to be inconsistent. TS reflects the system requirements for the location accuracy. If the requirements are low, which means that the system may need less computational complexity or it hopes the effective distance of the signal propagation model is longer, and then it's sensible to choose a lower TS value. If the system requires high accuracy, TS should be larger.

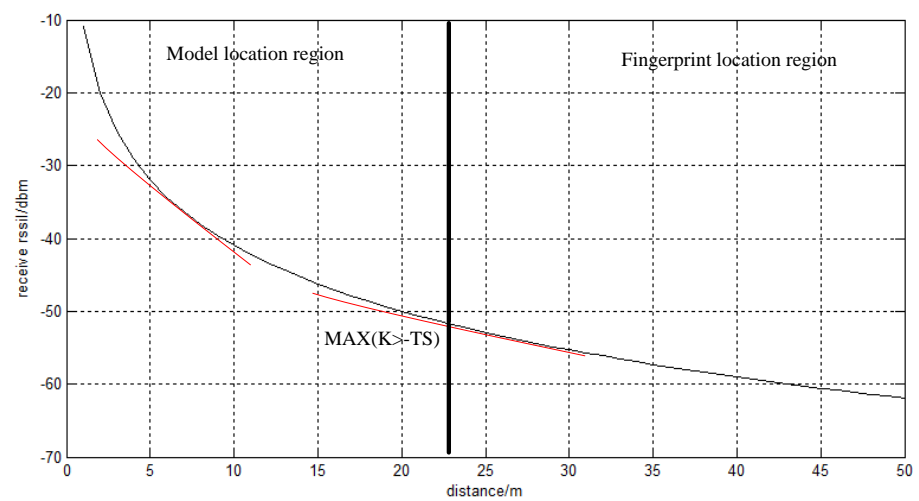

Figure 4. Calculate the Border of Signal Propagation Model

\section{Experiment Analysis}

Location network is shown in Figure 5.The location network consists of three kinds of devices, tags, readers and servers. A tag is mainly used to detect the signal of different positions. A reader receives tag's signal and then sends to the communication server to store through the signal collection client. The location server plays a role on executing the location algorithm. 


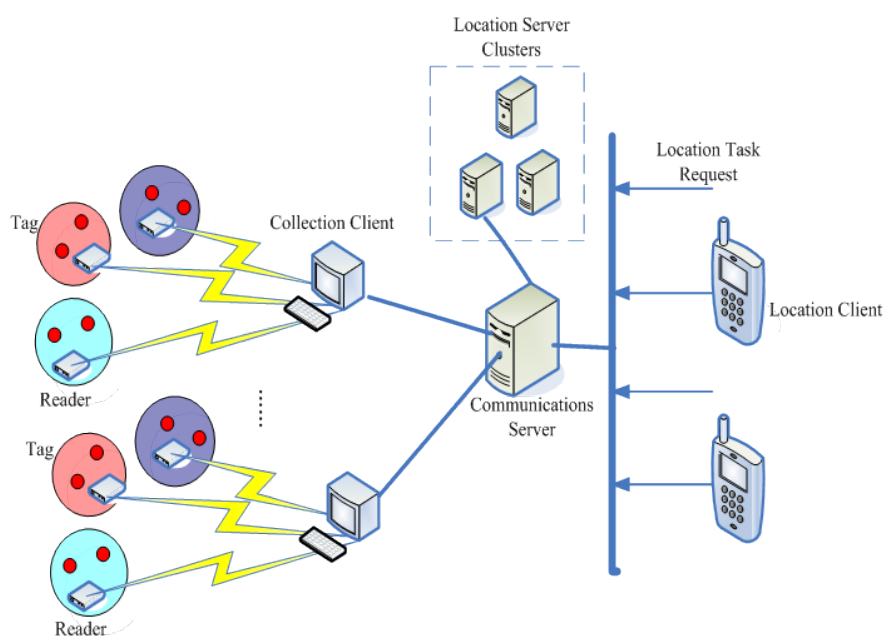

Figure 5. Localization Network

The experimental devices are shown in Figure 6. Tags and readers used in our system are provided by RFCode [13] Company. RFCode's active tags have the advantage in low-power and well-portability. The transmission distance of the facilities is up to $100 \mathrm{~m}$, and the reader is able to get the ID and RSSI from the tag. The M250 serial reader has 7 selectable frequency bands, and it can identify the RSSI value without conversion. In addition, the M250 reader has the function of wireless data transmission which supports some wireless communication protocols such as WiFi
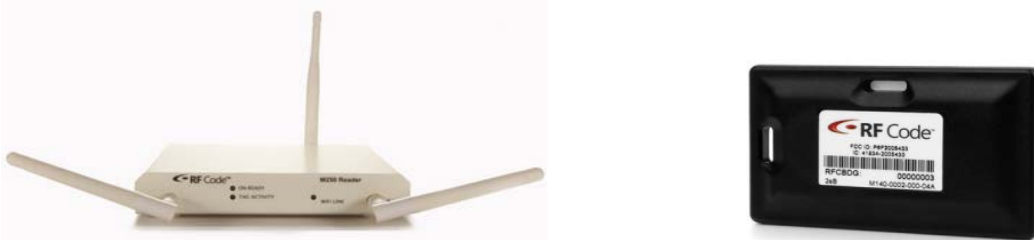

Figure 6. RFcode Reader and Active Tag

(1) Location experiments based on signal propagation model

The location experiment based on correction signal propagation model is conducted in the area of $5^{*} 15 \mathrm{~m}^{2}$, and the location result is shown in Figure 7 . The red circles stand for the real test points, and the triangles are the estimated positions calculated by signal propagation model. The mean error is about $1.6 \mathrm{~m}$. 


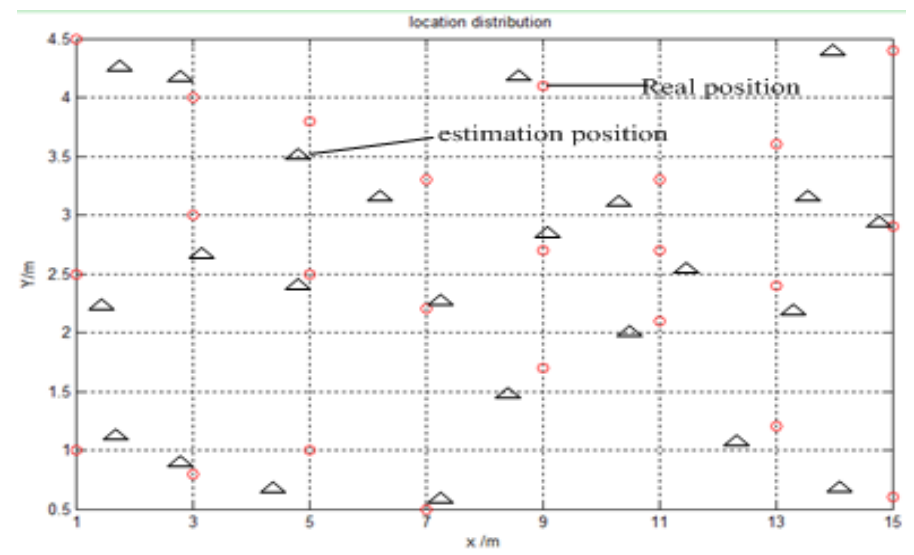

Figure 7. Location Result of Signal Propagation Model

(2) Location experiments based on fingerprinting method

To verify the fingerprinting-based location method, tests are conducted in the area of $5 * 10 \mathrm{~m}^{2}$. Firstly, 150 points are selected as the initial particles. In order to analyze the impact on location result among different training data sets, we choose 40 and 90 sample points in the offline phrase respectively. The data is collected 70 times at each point. The cumulative error distribution is shown in Figure 8. We can get the conclusion that the location accuracy increases with the number of particle. But the complexity in the online phrase will increase a lot. When the number of sample points is up to 90 , the mean error is about $1 \mathrm{~m}$.

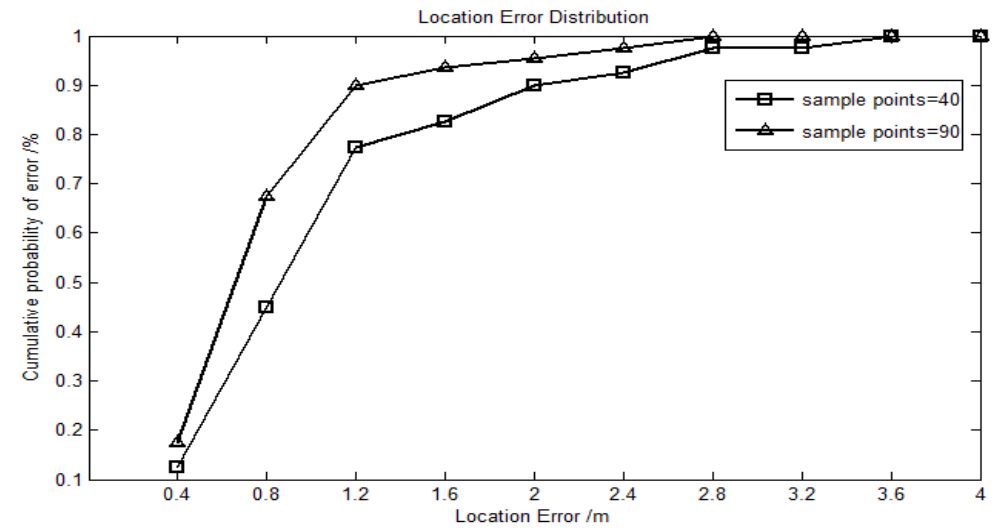

Figure 8. The Comparison in Different Numbers of Sample Point

(3) Experimental analysis of hybrid location approach

The comparative experiments of different location approaches are conducted in a large area of $5^{*} 30 \mathrm{~m}^{2}$ as shown in Figure 9. 


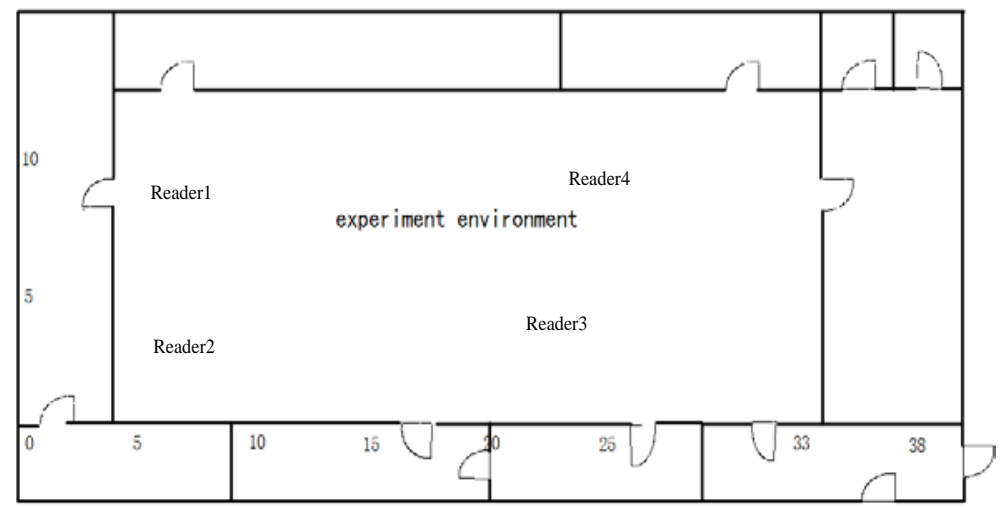

Figure 9. The Experiment Environment in Comparative Experiments

The experiments are divided into two groups and then we test the location orbits of two methods, the hybrid location and literature [14] respectively. In the hybrid location, we set the critical distance as $17 \mathrm{~m}$, and choose 90 sample points in fingerprinting location, as shown in Figure 10.

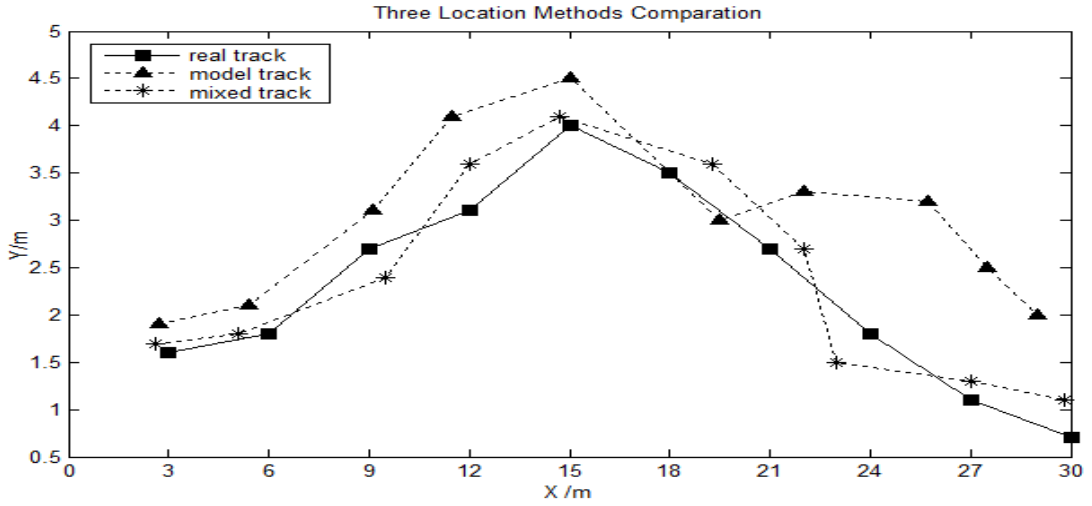

Figure 10. The Comparison between Model Tracking and Mixed Tracking

It is noticed that when the distance is within $17 \mathrm{~m}$, the location result based on signal propagation model is close to the real position. However, the location error gradually increases when the distance is greater than $17 \mathrm{~m}$. While the hybrid location approach is relatively stable, the mean error is about $1.3 \mathrm{~m}$. Thus, for a larger area, hybrid location approach has a certain advantage to avoid the complexity compared with just using fingerprinting location method, and it can also avoid the limitation of signal propagation model in external region. As long as signal propagation model estimation is accurate and the sample is reasonable, the hybrid location approach can get better results.

\section{Conclusions}

This paper proposes a hybrid location approach, and it makes full use of the advantage of signal propagation model and fingerprinting. The approach not only ensures the location accuracy, but also effectively improves the location speed. Indoor location is the key technology of the location-based services. The future research will focus on model self-adaptation to environment. Meanwhile, how to reduce the complexity and enhance the flexibility of the fingerprinting location are problems to be solved. 


\section{Acknowledgements}

This work was supported by a grant from the Natural Science Foundation of China (No. 61172018), the Science \& Research Plan Project of Shaanxi Province (No. 2011NXC01-12) and Science \& Research Plan Project of Shaanxi Province Department of Education (No.2010JC15). The authors are grateful for the anonymous reviewers who made constructive comments.

\section{References}

[1] Z. Jun, L. Hong-bin and W. Zhi, “An Active Queue Management Algorithm Based on Single Neuron Adaptive PI Controller”, The journal of Information and Control, vol. 37, no. 4, (2008), pp. 465-471.

[2] A. Harter and A. Hopper, “A distributed location system for the active office”, IEEE Network, vol. 8, no. 1, (1994), pp. 62-70.

[3] N. B. Priyantha, A. Chakraborty and H. Balakrishman, “The cricket location-support system”, Proceedings of the $6^{\text {th }}$ Annual International Conference on Mobile Computing and Networking, New York, USA, (2000).

[4] Y. W. Lee, E. Stuntebeck, S. C. Miller, "MERIT: Mesh of RF sensors for indoor tracking”, Processing of the $3^{\text {rd }}$ Annual IEEE Communication Society on Sensor and Ad Hoc Communications and Networks, Piscataway, USA, (2006).

[5] G. V. Zaruba, M. Huber, F. A. Kamangar and I. Chlamtac, "Monte Carlo sampling based in-home location tracking with minimal RF infrastructure requirements”, Proceeding of the IEEE Global Telecommunications Conference, Piscataway, USA, (2004).

[6] D. Madigam, E. Elnahrawy, R. P. Martin, J. Wen-Hua, P. Krishman and A. S. Krishnakumar, "Bayesian indoor positioning system", Proceeding of the $24^{\text {th }}$ Annual Joint Conference of the IEEE Computer and communications Societies, Piscataway, USA, (2005).

[7] L. Yiming, L. Haiyong, L. Jintao and Z. Fang, "Dynamic Radio Map Based Particle Filter for Indoor Wireless Location”, The Journal of Computer Research and Development, vol. 48, no. 1, (2011), pp. 139-146.

[8] G. Qing-hua, W. Jie, J. Ming-lu and W. Hong-yu, “An Indoor Location Method Based on Dynamic Radio Map”, Journal of Microwaves, vol. 26, no. 1, (2010), pp. 91-96.

[9] L. Quan, Z. Fang, L. Hai-Yong and K. Yi-Mei, “A Wireless Localization Algorithm Based on Spectral Decomposition of the Graph Laplacian”, The journal of Act Automatica SINICA, vol. 37, no. 3, (2011), pp. 316-321.

[10] L. M. Ni, Y. Liu, Y. Cho Lau and P. Abhishek, “LANDMARC: Indoor location sensing using active RFID”, Wireless Network, vol. 10, no. 6, (2004), pp. 701-710.

[11] P. Bahl and V. N. Padmanabhan, "RADAR: An in-building RF-based user location and tracking system”, Proceedings of the Nineteenth Annual Joint Conference of the IEEE Computer and Communications Societies, Piscataway, USA, (2000).

[12] Z. Fang, L. Hai-yong, L. Quan and M. Yan, "Node localization algorithm based on Kernel function and Markov chains”, Journal on Communications, vol. 31, no. 11, (2010), pp. 195-204.

[13] RF Code, Inc., http://www.rfcode.com.

[14] C. Chang-xiang, D. Wei and Z. Jie, "RSSI-based Range Collection Localization Algorithm in WSN", Communications Technology, vol. 44, no. 2, (2011), pp. 67-69.

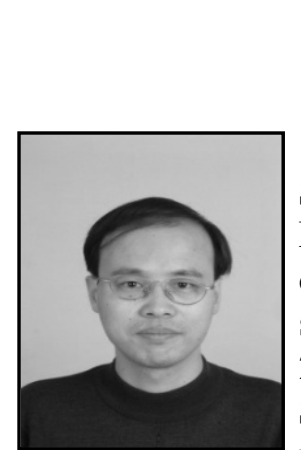

\section{Authors}

Junhuai Li received the B.S. degree in electrical automation from Shaanxi Institute of Mechanical Engineering of China, Xi' an in 1992, M.S. degree in computer application technology from Xi'an University of Technology of China, Xi'an in 1999, and Ph.D. degree in computer software and theory from Northwest University of China, Xi'an in 2002. He was in University of Tsukuba of Japan from March to September in 2004 as a visiting scholar. He is currently a professor with School of Computer Science and Engineering, Xi'an University of Technology, China. His research interests include Internet of Things technology, network computing. 

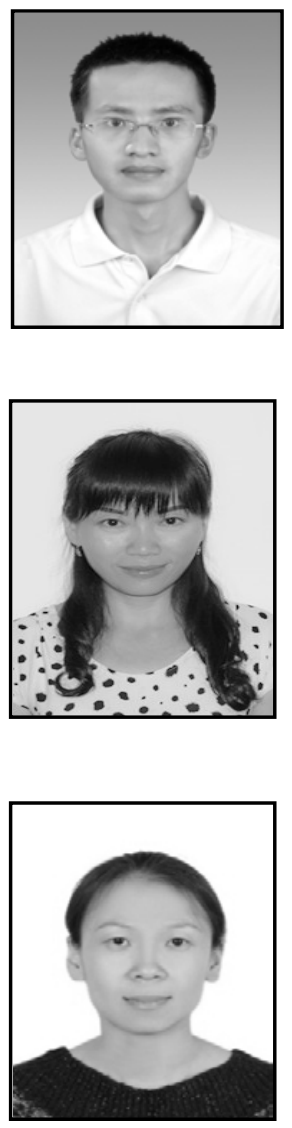

Lei Yu received her MS degree in Computer Science from Xi'an University of Technology, Xi'an, China, in 2003; She is currently a lecturer in faculty of computer science and engineer of XAUT. Her research interests are in RFID and data mining.

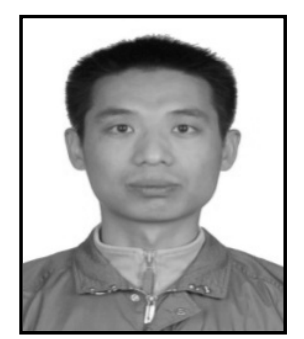

Zhixiao Wang received the B.S. degree from Shijiazhuang Economic University of China in 2000 and the M.S. degree from Xi'an University of Technology of China in 2004, all in computer science. $\mathrm{He}$ is a PhD candidate in the Department of Computer Science at Xi'an Jiaotong University, China. His current research interests include wireless and mobile networks. 